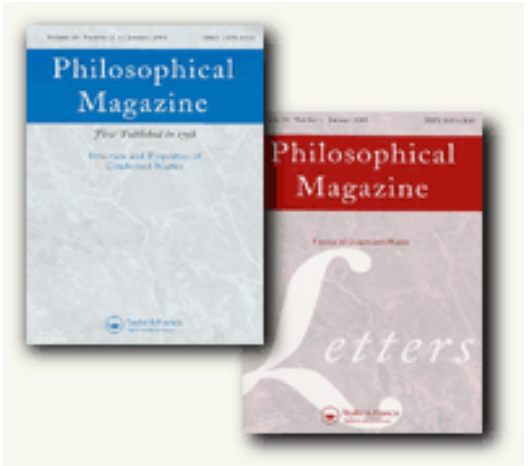

\title{
Stress and strain around grain-boundary dislocations measured by high-resolution electron microscopy
}

\begin{tabular}{|c|c|}
\hline Journal: & Philosophical Magazine \& Philosophical Magazine Letters \\
\hline Manuscript ID: & TPHM-05-Dec-0557.R2 \\
\hline Journal Selection: & Philosophical Magazine \\
\hline $\begin{array}{r}\text { Date Submitted by the } \\
\text { Author: }\end{array}$ & 23-Mar-2006 \\
\hline Complete List of Authors: & $\begin{array}{l}\text { HYTCH, Martin; CEMES-CNRS } \\
\text { Putaux, Jean-Luc; CERMAV-CNRS } \\
\text { Thibault, Jany; TECSEN-CNRS }\end{array}$ \\
\hline Keywords: & $\begin{array}{l}\text { dislocations, grain boundaries, high-resolution imaging, image } \\
\text { analysis, stress-strain measurements }\end{array}$ \\
\hline Keywords (user supplied): & \\
\hline
\end{tabular}

\section{\$) ScholarONE \\ Manuscript Central}




\title{
Stress and strain around grain-boundary dislocations measured by high-resolution electron microscopy
}

\author{
M. J. HŸTCH ${ }^{1}$, J.-L. PUTAUX ${ }^{2}$ AND J. THIBAULT ${ }^{3}$
}

1 CEMES-CNRS, 29 rue J. Marvig, 31055 Toulouse, France

2 CERMAV-CNRS, BP 53, 38041 Grenoble, France. Affiliated with the Joseph Fourier University of Grenoble.

3 TECSEN-UMR-CNRS 6122, Université Aix-Marseille III Paul Cézanne, Faculté des Sciences et Techniques de St Jérôme, 13397 Marseille, France.

Correspondence should be addressed to M.J. Hÿtch (e-mail: hytch@cemes.fr).

\begin{abstract}
Stresses and strains around a dislocation at a grain boundary in germanium are measured by a combination of high-resolution electron microscopy and geometric phase analysis. The method is established by first measuring the strains around a matrix dislocation in silicon. Stresses are determined using linear elastic theory and bulk elastic constants. Strain measurements are shown to agree with theoretical calculations based on linear anisotropic elastic theory to $0.2 \%$ at a spatial resolution of $2-3 \mathrm{~nm}$. A dislocation constricted at a coherent twin boundary in germanium is subsequently analysed. The method is adapted to cope with the problem that the reference lattice is not identical for the whole field of view, due to the grain boundary. Strains are compared with theoretical calculations of a matrix dislocation in germanium. Whilst strains in the grains on either side of the twin boundary agree closely with the isolated dislocation case, significant additional strains are localised at the boundary plane. By comparing the stresses and strains across the boundary plane, values for the elastic modulus of the twin boundary are proposed. The significant reduction in elastic modulus for
\end{abstract}


the boundary, when compared to bulk elastic constants, is interpreted in terms of the nonequilibrium configuration of the boundary. An extension of the method is proposed to measure more generally the elastic properties of grain boundaries and interfaces.

\section{Introduction}

The strain field around isolated dislocations has been studied in detail within the framework of linear elastic theory [1]. Recently it has been shown that even at the nanoscale, theoretical predictions and experimental measurements of the displacement field agree remarkably well [2]. The strain around dislocations located at grain boundaries and interfaces is less well established, mainly due to the analytical and conceptual difficulties involved. An exception concerns low-angle grain boundaries which can be considered as an array of individual dislocations [3]. Here again, elastic theory and experimental measurements are in very close agreement, particularly for the rotation field [4]. The strain at more general heterophase boundaries containing interfacial dislocations has been treated within elasticity theory as a bimaterial of two joined half-spaces, each having the elastic properties of the bulk [5]. However, there are very good reasons to suppose that elastically the boundary will behave differently to the surrounding matrix, if only from the fact that the local atomic configurations and bonding are specific to the boundary plane.

The concept and definition of effective elastic properties for grain boundaries has been developed in continuum elastic theory [6]. The full stiffness tensor can then be calculated using atomistic modelling for different grain boundary structures [7,8]. For metals it was shown that elastic moduli can differ quite significantly from the bulk, particularly for energetically unfavourable boundary configurations. The ultimate aim is to improve mesoscopic modelling of mechanical straining of materials containing grain boundaries. Compatibility conditions for the stresses and strains across boundaries have been elaborated 
[9] and used for modelling the strain response of twin boundaries for example [10]. For the moment, however, only theoretical results have been presented, the most relevant to the current study concerning twin boundaries in silicon [11].

Here we will present the measurement of stress and strain around dislocations at grain boundaries by a combination of high-resolution electron microscopy (HREM) and geometric phase analysis [12]. The usual limitations to this technique will apply, in that measurements are restricted to in-plane components and exterior to the dislocation core. Stresses will be determined by applying linear elastic theory to the measured strains [1]. We will begin, in order to establish the technique, with the presumably well known case of an isolated dislocation in the matrix. This will allow us to study the more interesting case of a dislocation located at a grain boundary. The strains will be shown to be different to the isolated dislocation case and hence that the grain boundary influences the local stresses and strains. By analysing the stresses and strains, we will show that the specific elastic properties of grain boundaries can be determined.

\section{Experimental methods}

\subsection{Geometric phase analysis}

Geometric phase analysis (GPA) is an image-processing routine that is sensitive to small displacements of the lattice fringes in HRTEM images relative to a reference lattice [12]. Displacements are measured by analyzing the local Fourier components of the lattice fringes, g, in an image by Fourier filtering. The size of the mask used in the filtering will determine the spatial resolution of the results obtained. The resulting phase image, $\mathrm{P}_{\mathrm{g}}(\mathbf{r})$, describes the positions of the lattice fringes in real space. Any displacement of the lattice fringes with respect to the reference will result in a phase shift, i.e., a change in the value of the phase at the position corresponding to the displacement. Accordingly, the phase image is described as: 


$$
P_{g}(\mathbf{r})=-2 \pi \mathbf{g} \cdot \mathbf{u}(\mathbf{r})
$$

where $\mathbf{u}(\mathbf{r})$ is the local displacement with respect to the reference lattice, $\mathbf{g}$. An identical phase component appears in the Howie-Whelan equations to include dynamical scattering from a local lattice distortion [13]. An individual phase image gives only the component of the displacement field in the direction of $\mathbf{g}$. Two phase images, $\mathrm{P}_{\mathrm{g} 1}$ and $\mathrm{P}_{\mathrm{g} 2}$ (where $\mathbf{g}_{1}$ and $\mathbf{g}_{2}$ are non-colinear), are required to determine the two-dimensional displacement field, $\mathbf{u}(\mathbf{r})$ :

$$
\mathbf{u}(\mathbf{r})=-\frac{1}{2 \pi}\left[P_{g 1}(\mathbf{r}) \mathbf{a}_{1}+P_{g 2}(\mathbf{r}) \mathbf{a}_{2}\right]
$$

where $\mathbf{a}_{1}$ and $\mathbf{a}_{2}$ are the real-space basis vectors corresponding to the reciprocal lattice defined by $\mathbf{g}_{1}$ and $\mathbf{g}_{2}$ [12]. The strain tensor can then be obtained by numerical differentiation using the standard relations [1]:

$$
\varepsilon_{i j}=\frac{1}{2}\left(\frac{\partial u_{i}}{\partial x_{j}}+\frac{\partial u_{j}}{\partial x_{i}}\right)
$$

In a similar way the local in-plane rigid body rotation, $\omega_{\mathrm{xy}}$, can be determined:

$$
\omega_{x y}=\frac{1}{2}\left(\frac{\partial u_{y}}{\partial x_{x}}-\frac{\partial u_{x}}{\partial x_{y}}\right)
$$

where, for small rotations, the angle is in radians and anti-clockwise positive. The more developed calculations for large deformations are not necessary here (see Appendix E in [12]) though are implemented in the practice.

Assuming that linear elastic theory is valid at the nanoscale, the stresses can be determined from the deformation [1]: 


$$
\left[\begin{array}{l}
\sigma_{11} \\
\sigma_{22} \\
\sigma_{33} \\
\sigma_{12}
\end{array}\right]=\left[\begin{array}{llll}
c_{11}^{\prime} & c_{12}^{\prime} & c_{13}^{\prime} & c_{16}^{\prime} \\
c_{12}^{\prime} & c_{22}^{\prime} & c_{23}^{\prime} & c_{26}^{\prime} \\
c_{13}^{\prime} & c_{23}^{\prime} & c_{33}^{\prime} & c_{36}^{\prime} \\
c_{16}^{\prime} & c_{26}^{\prime} & c_{36}^{\prime} & c_{66}^{\prime}
\end{array}\right]\left[\begin{array}{c}
\varepsilon_{11} \\
\varepsilon_{22} \\
\varepsilon_{33} \\
2 \varepsilon_{12}
\end{array}\right]
$$

where $\mathrm{c}^{\prime}{ }_{\mathrm{ij}}$ are the components of the elasticity stiffness tensor in the coordinates of the image $x y$-plane ( 1 and 2 corresponding to the $x$ and $y$-axes respectively and the third axis corresponding to the viewing direction). The other components have been omitted as these are not measurable by in-plane analysis. The strain in the observation direction, $\varepsilon_{33}$, has been included, however, since for plane strain conditions it can be assigned a value, i.e. zero.

\subsection{Electron microscopy}

Electron microscopy was carried out on $\Sigma 9(122)$ and $\Sigma 3(111)$ bicrystals respectively of silicon and germanium. Macroscopic stresses were applied in order for the bicrystal to remain compatible [14]. Dislocations are generated in both grains. In the $\Sigma 9(122)$ two glide systems are generally activated in each grain. If grain I is considered, the Burgers vectors are either $1 / 2[\overline{1} 01]^{I}$ and $1 / 2[011]^{I}$, for dislocations gliding on $(11 \overline{1})^{I}$ planes, or $1 / 2[10 \overline{1}]^{I}$ and $1 / 2[01 \overline{1}]^{I}$, for dislocations gliding on $(111)^{I}$ planes. Some of these dislocations combine to form sessile Lomer-Cottrell dislocations of Burgers vector $1 / 2[110]$. Two types of dislocations will be studied in the following: one isolated Lomer dislocation in $\mathrm{Si}$ and one $60^{\circ}$ dislocation stopped at the $\Sigma 3(111)$ twin boundary in Ge. Thin foils were prepared by mechanical polishing to a thickness of about $70 \mu \mathrm{m}$ followed by ion milling in a Gatan duoMill at $6 \mathrm{kV}$. The amorphous surface layer was removed by chemical etching in a $\mathrm{HF} 10 \%-\mathrm{HNO}_{3} 90 \%$ solution at $0^{\circ} \mathrm{C}$. Images were taken at the common [1 T0] zone axis on a JEOL 200CX operating at $200 \mathrm{kV}(\mathrm{Cs}=1.1 \mathrm{~mm}$, point resolution $0.22 \mathrm{~nm})$ and the negatives digitised at $0.0386 \mathrm{~nm}$ per pixel. Despite the age of the negatives, physical distortions were negligible in the relatively small area of film analysed. Geometric Phase Analysis (GPA) was performed using the plug- 
in GPA Phase 1.0 (HREM Research) [15] for the software package DigitalMicrograph 3.9+ (Gatan). Lattice fringes were analysed by applying Lorentzian masks in reciprocal space of radius $0.4 \mathrm{~nm}^{-1}$ producing a lateral resolution in the phase images of $2.5 \mathrm{~nm}$.

\section{Matrix dislocation in silicon}

Figure 1 (a) shows a high-resolution image of a pure edge Lomer dislocation in silicon with Burgers vector $\mathbf{b}=1 / 2[110]$ seen end-on in [1 $\overline{1} 0]$ orientation. The uniform contrast, showing little variation in the amplitude of the lattice fringes, is ideal for phase analysis [16]. This image was previously analysed to determine the displacement field to picometre accuracy at nanometre scale lateral resolution [2]. Indeed, in the area between 5 and $10 \mathrm{~nm}$ from the dislocation core, experimental and theoretical values agree to within $3 \mathrm{pm}$. Here, the aim is measure the stress and strain so a smaller mask was used in reciprocal space to further improve the signal-to-noise ratio: strain is particularly sensitive to noise as it is calculated by derivative of the displacement, which always amplifies random fluctuations. Phase images were calculated for the (111) and the $(11 \overline{1})$ lattice fringes. The phases were then converted using Equation (2), into the displacement parallel (x-axis) and perpendicular (y-axis) to the Burgers vector (Figure 1 (b)).

The strain field can be determined by numerical differentiation of the displacement field, according to Equation (3). In practice, derivatives are taken directly from the phase images and then combined. The results for the experimental images are given in Figure 2 (ac). Contours have been added to maximum strains of $\pm 2.5 \%$. Larger values of strain occur in the immediate core region but their interpretation is problematic: objective lens aberrations will certainly produce image artifacts in this region. In order to compare the results with theory, the displacement field was calculated using anisotropic elastic theory for a dislocation in an infinite medium and using the bulk elastic constants of silicon [1]. Theoretical phase 
images for the (111) and (11 $\overline{1})$ lattice fringes were calculated using Equation (1) so that identical routines to the experimental case could be used to determine the strains (Figure 2).

At this point, a word should be said about the choice of the reference lattice. Measurement of displacement and deformation are always relative to a particular lattice. In addition, strain in a mechanical sense is defined with respect to the undeformed state. From current high-resolution work, it is not possible to know the state of the specimen prior to the introduction of a dislocation, or indeed before the making of the thin foil. The first approximation is to choose a region of crystal at some distance from the defect, and to assume that the lattice is undeformed there. At $10 \mathrm{~nm}$ from the dislocation core in the direction perpendicular to the Burgers vector, all the strain components are theoretically below $0.2 \%$, and serves as a good first choice for the reference. As a refinement, the strain field has a certain symmetry theoretically. The reference was adjusted slightly to account for this fact. In any case, the strain fields change very little: the distribution changes not at all, only in the absolute mean level. A global dilatation, or rotation, of the crystal in the reference area due to the presence of a dislocation cannot therefore be detected using this current method. Displacement fields are much more sensitive in appearance, as a change in the reference produces a ramp in the displacement field, integrating any changes across the whole field of view, which can be quite considerable. The physically significant field is of course the strain, as this produces the actual forces between atoms.

With the chosen reference, the experimental and theoretical distributions can be seen to agree very well. Notice in particular the butterfly shape of the principal strain component, $\varepsilon_{\mathrm{xx}}$, parallel to the Burgers vector. In the region of the extra half-plane $(\mathrm{y}>0)$, the strains are negative and compressive, and on the other side the lattice is in expansion. The strain perpendicular to the Burgers vector, $\varepsilon_{\mathrm{yy}}$, forms a characteristic three-fold symmetry, whilst the shear component, $\varepsilon_{\mathrm{yy}}$, is mainly concentrated in the glide plane. 
Also included, Figure 2 (d), are the results for the local rigid-body rotation, calculated via Equation (4), and forms two lobes of negative and positive rotations. The lobe axis is parallel to the Burgers vector, the negative values representing the bending of the lattice to the right, making way for the extra half-plane inserted along the positive y-axis. In a similar way, the lattice bends in the clockwise direction to the right of the half-plane. Indeed, the rotation field can be shown to have a particularly simple form in isotropic elastic theory, independent of materials parameters $[4,17]$ :

$$
\omega_{x y}=-\frac{b}{2 \pi r} \cos \theta
$$

where the polar coordinates $(r, \theta)$ are with respect to an $x$-axis parallel to the Burgers vector.

Linear elastic theory can be used to calculate values for the stresses. Assuming plane strain conditions for this edge dislocation, Equation (5) becomes:

$$
\left[\begin{array}{l}
\sigma_{11} \\
\sigma_{22} \\
\sigma_{33} \\
\sigma_{12}
\end{array}\right]=\left[\begin{array}{cccc}
c_{11}^{\prime} & c_{12}^{\prime} & c_{13}^{\prime} & 0 \\
c_{12}^{\prime} & c_{22}^{\prime} & c_{23}^{\prime} & 0 \\
c_{13}^{\prime} & c_{23}^{\prime} & c_{33}^{\prime} & 0 \\
0 & 0 & 0 & c_{66}^{\prime}
\end{array}\right]\left[\begin{array}{c}
\varepsilon_{11} \\
\varepsilon_{22} \\
0 \\
2 \varepsilon_{12}
\end{array}\right]
$$

where the values for the stiffness tensor are given in Table 1 for an $x$-axis parallel to [110]. The results are given in Figure 3. Contours have been limited to the values appearing in the immediate vicinity of the core where the results are more than doubly uncertain as linear elastic theory is only valid for small deformations, and is based on a continuum theory ignoring the existence of discrete atoms.

The use of the plane strain solution receives two justifications. Firstly, the stresses perpendicular to the foil normal are relatively weak (see Figure 3 (d)) and highly localized at the dislocation core. Thin film relaxation depends on the ratio of the lateral extent of the stress field and the foil thickness [18]. For example, for sinusoidal stress fields, it is the ratio of the 
wavelength and the foil thickness which imports: large values indicate conditions close to plane stress solutions, small values plane strain and intermediate values uncertain effects. For an estimated core width of the order of $1 \mathrm{~nm}$ and a foil thickness of $20 \mathrm{~nm}$, plane strain solution should be accurate. The second justification, and the most valid, comes from the excellent match between experimental and theoretical distributions for each of the stress and strain tensor components.

To analysis the degree of agreement between experiment and theory in more detail, the largest strain component, $\varepsilon_{\mathrm{xx}}$, was subtracted from the theoretical strain field (Figure 4). In the circular region between 5 and $10 \mathrm{~nm}$ from the dislocation core, the standard deviation of the residual is only $0.2 \%$. This is our estimation of accuracy of the technique. In this region, the residual has random oscillations on a length scale characteristic of the spatial resolution of the technique. To understand better the scale of the measurements, the principal stress component is superimposed on the original high-resolution image in Figure 5. The spatial resolution of the results is indeed on the scale of $2-3 \mathrm{~nm}$, as indicated by the size of the mask used in the analysis, if judged by the meanders in the contours. Figure 5 is redolent of the diffraction contrast images used to reveal the strain component $\varepsilon_{\mathrm{Xx}}$ around an edge dislocation seen endon [19].

Returning to the strain residual (Figure 4), measurable differences do exist in the core region. It is also intriguing that these systematic differences, albeit small, are present several nanometres from the core. The 3 -fold symmetry is similar to the $\varepsilon_{\mathrm{yy}}$ strain field and could mean that the dislocation core is slightly dissociated as expected from previous work [20]. However, experimental strain fields are compared directly with theoretical elastic fields and not with those extracted from simulated images of the dislocation core, which represents an obvious source of error. The effect of averaging during the image processing cannot be 
excluded either. Interesting as these results are, only extensive simulation and analysis will help further their interpretation.

\section{A grain-boundary dislocation in germanium}

Figure 6 shows the interesting case of a lattice dislocation constricted at the twin boundary in the germanium bicrystal. The edge component of the Burgers vector was determined using the grain-boundary circuit mapping [21] to be $1 / 4[112]^{I}$ corresponding to the edge component of a $60^{\circ}$ matrix dislocation of total Burgers vector $1 / 2[101]^{I}$, or $1 / 2[011]^{I}$ depending on the sign of the screw component. It appears that the dislocation has not yet had the time (or the energy) to decompose into a sessile part $1 / 3[111]^{I}$ and glissile part $1 / 6[121]^{I}$ (or $1 / 6[211]^{I}$ ), as is usually the case. The grain boundary is therefore in a non-equilibrium configuration.

Displacements of the lattice perpendicular to the twin boundary plane, $\mathrm{u}_{\mathrm{x}}$, were measured by GPA by calculating the phase of the $(111)^{\mathrm{I}} / /(11 \overline{1})^{\mathrm{II}}$ lattice fringes common to both crystals. Measurement of displacements parallel to the boundary, $\mathrm{u}_{\mathrm{y}}$, were more problematic as the other set of $\{111\}$ fringes are not common to both crystals, due to the twin. Displacements were therefore measured separately in each grain I \& II using references:

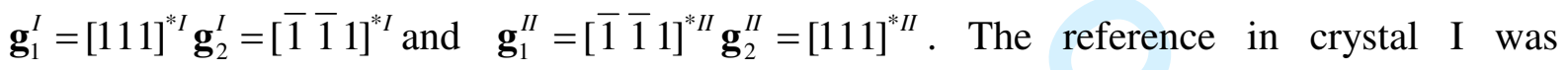
determined numerically from the image and then, using the orientation relation for a perfect twin, the reference state in crystal II was determined according to: $\mathbf{g}_{1}^{I I}=-\mathbf{g}_{1}^{I}, \mathbf{g}_{2}^{I I}=\mathbf{g}_{2}^{I}+\frac{2}{3} \mathbf{g}_{1}^{I}$. This makes the procedure similar to that in circuit mapping [21]. Strains, $\varepsilon_{\mathrm{ij}}$, and local rigidbody rotations, $\omega_{\mathrm{xy}}$, were computed using Equation (4) in each crystal separately. The position of the interface was then determined from the original HRTEM image, dividing the field of view into two halves. The results for grain I were placed in the half corresponding to grain I 
and the results for grain II in the other half (procedure of course unnecessary for the $\varepsilon_{\mathrm{xx}}$ component). The results of this juxtaposition can be seen in Figure 7.

The concentration of strains at the centre of the image corresponds to the dislocation core, whose localisation confirms the picture of the $60^{\circ}$ dislocation constricted in the boundary plane. Strains are distributed in both crystals, as to be expected for a coherent boundary coupled elastically. In the major strain component, $\varepsilon_{\mathrm{xx}}$, the lattice is in compression above the dislocation, to accommodate the extra half-plane, and below in expansion, as seen previously for the matrix dislocation in silicon. The most interesting feature, however, is the localisation of the strains at the boundary plane. A spike of increased compression, with respect to the matrix, is apparent above the dislocation along the plane of the boundary, with a similarly increased expansion below. This is unlike the previous case shown for silicon and will be the subject of detailed analysis later on.

In order to analyse the similarities and dissimilarities with a matrix dislocation more directly, it is instructive to compare the experimental strain field with the theoretical strain field of a matrix dislocation in germanium. Figures 7 (a-c) shows the strains corresponding to an isolated, and non-dissociated, $60^{\circ}$ dislocation according to isotropic elastic theory. Although germanium is slightly anisotropic $(\mathrm{A}=1.66)$, isotropic theory proves sufficient for our present purposes (Poisson's constant $v=0.20$ for germanium [1]).

The general features of the strain field are well reproduced, including the slight dissymmetry of the compressive and expansive lobes due to the inclination of the Burgers vector to the horizontal plane. The strain component, $\varepsilon_{\mathrm{yy}}$, parallel to the boundary plane (Figure 7 (b)) is continuous across the boundary and shows no localised strains. The slight discontinuity at the twin is due to the piece-wise calculation of the strains (notably absent from $\varepsilon_{\mathrm{xx}}$ due to the common lattice planes parallel to the boundary). Indeed, the continuity of 
$\varepsilon_{\mathrm{yy}}$ is a necessary condition for elastic equilibrium [9]. We have used this condition to refine the y-component of the reference lattice, analogue to the previous case of the matrix dislocation. Adjusting the reference only adds a constant value to the strain components, so it is gratifying to see that the variation of $\varepsilon_{\mathrm{yy}}$ matches extremely well along the boundary plane and can be taken as a useful validation of the results.

A discontinuity is possible for the shear component, $\varepsilon_{\mathrm{xy}}$, at the boundary [9]. However, the slight discontinuity above the dislocation is most probably due to an artefact from the image analysis. The contrast of the lattice fringes needed for the analysis goes to zero at the boundary plane, a highly discontinuous occurrence which can produce artificial strains when taking derivatives in this direction (necessary for $\varepsilon_{\mathrm{xy}}$, but not $\varepsilon_{\mathrm{yy}}$ ) [16]. The rigid-body rotation shows the direction of the Burgers vector in perhaps the clearest fashion - the lobes are almost exactly parallel to the direction [112] $]^{\mathrm{I}}$. The fact that the theoretical contours are circular comes from the use of isotropic elastic theory (Equation (6)), rather than the anisotropic theory used for the silicon dislocation.

It seems therefore that the effect of the twin boundary is manifest principally in the strain perpendicular to the boundary plane, $\varepsilon_{\mathrm{xx}}$. The other components resemble closely the isolated dislocation case. The major difference is along the boundary plane, as can be seen clearly in the difference image (Figure 8) calculated by subtracting the experimental and theoretical strains. To the left and right of the boundary plane in the matrix area of grains I and II, the residual does not show any particular systematic variations, only random fluctuations. Judging from the standard deviation of the difference image and the length scale of variations in the matrix region, an estimate for the accuracy of the strain measurements can be obtained of $0.2 \%$ at a spatial resolution of $2-3 \mathrm{~nm}$. However, along the boundary plane there is a clear increase in the compression (with respect to theory) above the dislocation and an increased expansion below. The localisation of these strains can be better appreciated in $-12-$ 
the composite image in Figure 9. The strains do not coincide exactly on the grain boundary position seen in the HRTEM lattice image but it must be remembered that the strain measurements have a certain spatial resolution, in this case about $2.5 \mathrm{~nm}$. Noise will shift contours on this length scale.

\section{Analysis and discussion}

An explanation needs to be found for the behaviour of the strain field. For the highly symmetric twin boundary and assuming a simple bimaterial model, the strain field would not be expected to differ greatly from the isolated dislocation solution, even taking crystalline anisotropy into account. Strains parallel to the electron beam direction, $\varepsilon_{\mathrm{zz}}$, are assumed to be zero, and that plane-strain conditions apply. The screw component of the dislocation will produce strains of the type $\varepsilon_{\mathrm{xz}}$ and $\varepsilon_{\mathrm{yz}}$. However, these will not affect in-plane stress components.

It is extremely unlikely that the expansion and contraction measured across the boundary plane is due to imaging artefacts or to rigid-body displacements induced by structural changes in the twin boundary. Either of these effects would tend to produce a uniform expansion (or contraction) of the boundary and there could be no reason for a reversal of sign above and below the dislocation. For example, dynamic scattering will certainly modify the apparent position of lattice planes at the boundary plane but from symmetry arguments the net effect should not produce overall contractions and expansions. Three-fold astigmatism can produce artificial rigid-body displacements at grain boundaries [22] - but only for non-common lattice planes, in our case in the y-direction, not for the common $\{111\}$ lattice planes perpendicular to the boundary.

It seems that the grain boundary does indeed behave differently elastically to the matrix $[6,7,8]$. As a simplification to the full treatment, we will imagine that a thin slab of 
material along the boundary has different elastic moduli to the material on either side (Figure 10). We can investigate its elastic properties by determining the stresses next to the boundary plane. For this we have extracted strain profiles parallel to the boundary as a function of distance from the dislocation core (see Figure 11 (a) for the profile in $\varepsilon_{\mathrm{xx}}$ ). The increase of strain at the boundary compared with the surrounding matrix is clearly visible. From these profiles, stresses have been determined using anisotropic elastic theory and the bulk elastic constants of germanium tabulated in Table 2 (see Figure 11 (b) for the profile of $\sigma_{11}$ ):

$$
\left[\begin{array}{l}
\sigma_{11} \\
\sigma_{22} \\
\sigma_{12}
\end{array}\right]=\left[\begin{array}{lll}
c_{11}^{\prime} & c_{12}^{\prime} & c_{16}^{\prime} \\
c_{12}^{\prime} & c_{22}^{\prime} & c_{26}^{\prime} \\
c_{16}^{\prime} & c_{26}^{\prime} & c_{66}^{\prime}
\end{array}\right]\left[\begin{array}{c}
\varepsilon_{11} \\
\varepsilon_{22} \\
2 \varepsilon_{12}
\end{array}\right]
$$

For elastic equilibrium, the stress calculated in the matrix, $\sigma_{x x}$, should equal the stress at the boundary, $\sigma_{x x}^{g b}$, and then:

$$
\sigma_{x x}=\sigma_{x x}^{g b}=c_{11}^{g b} \varepsilon_{x x}^{g b}+c_{22}^{g b} \varepsilon_{y y}^{g b}+c_{16}^{g b} \varepsilon_{x y}^{g b}
$$

where $c_{i j}^{g b}$ are the effective elastic constants of the twin boundary. There are three unknowns in this equation. However, the dominant term is the first, and neglecting the second and third terms on the right hand side, an estimate for the expansion stiffness of the boundary can be found:

$$
c_{11}^{g b} \approx \sigma_{x x} / \varepsilon_{x x}^{g b}
$$

Analysing the numerical values determined from Equation (10) between 5 and $10 \mathrm{~nm}$ from the core, we find that the grain boundary stiffness is $100 \pm 15 \mathrm{GPa}$ (mean and standard deviation) above the dislocation and $70 \pm 25 \mathrm{GPa}$ below, and on average $85 \pm 30 \mathrm{GPa}$. This is to be compared with the value of $165 \mathrm{GPa}$ in the matrix. 
The values obtained for the grain-boundary stiffness in the compressed and expanded regions of the boundary are close but not identical. The main difference comes from the fact that the matrix seems more strained in the expanded part than in the compressed (see Figure 11 (a)). Whilst the compressed curve follows closely elastic theory, the expanded part is higher. This can either indicate that isotropic theory is indeed inadequate, or that the strains measured in the matrix are an overestimate of the values immediately next to the grain boundary. Matrix strains were measured at $3 \mathrm{~nm}$ from the boundary because of the limited spatial resolution of the measurements of $2-3 \mathrm{~nm}$. Using the strains predicted by elastic theory would produce values for the boundary stiffness of $70 \pm 15 \mathrm{GPa}$, in complete accordance to the compressive region.

Values of this order have been found from atomistic simulations of incoherent twins in silicon [11]. Simulations show that elastic moduli are sensitive to the exact boundary configuration and high-energy boundaries tend to be less stable elastically. A general feature of the calculations is that elastic properties rapidly attain bulk values a few unit cells from the boundary, i.e. 1-2 nm in our case. Given the spatial resolution of our measurements it is not possible to verify whether this is true or not. We probably detect the strain at the boundary itself. Unfortunately, some of the biggest effects are witnessed theoretically for the shear moduli for which we have no estimates yet. Further analysis therefore requires calculations to be performed with the exact experimental atomic boundary structure and possibly with higher spatial resolution.

\section{Conclusions}

Experimental methods to measure the elastic modulus of a grain boundary are rare [23] and mechanical testing of bi-crystals has been limited to yield and fracture stresses due to the difficulties of the experiments [24]. The analysis presented here could form the basis of a 
method of measuring grain-boundary elastic properties more directly. For example, in an ideal experiment, a dislocation could be brought into close proximity with the boundary so as to provoke measurable strains but not so as to modify the boundary structure. Images would need to be of the highest quality in order to have sufficient precision in the measurement of stresses and strains. In this respect, carrying out experiments with an objective lens aberration corrected machine would have obvious advantages [25].

Here, a coherent twin boundary containing a dislocation has been found to be significantly weaker than the surrounding crystal, by as much as $50 \%$. We believe this to be the case because the boundary is in a non-equilibrium configuration, and not that coherent twins are in general weaker. Future measurements could be carried out for many interesting configurations, grain boundaries and interfaces in natural and man-made materials.

\section{Acknowledgements}

The authors would particularly like to thank Jean-Michel Pénisson for many fruitful discussions. Part of this work was carried out in the CNRS funded European network (GDRE) "Quantification and measurement in transmission electron microscopy" regrouping laboratories in France, the United Kingdom, Germany and Switzerland.

\section{References}

[1] J.P. Hirth and J. Lothe, Theory of Dislocations: second edition (Krieger, Malabar Florida, 1992).

[2] M.J. Hÿtch, J.-L. Putaux and J.-M. Pénisson, Nature 423270 (2003).

[3] W.T. Read and W. Shockley, Phys. Rev. 78275 (1950).

[4] C.L. Johnson, M.J. Hÿtch and P. Buseck, PNAS 10117936 (2004).

$$
-16 \text { - }
$$


[5] R. Bonnet and M. Loubradou, Phys. Rev. B 4914397 (1994).

[6] I. Alber, J.L. Bassani, M. Khantha, V. Vitek and G.J. Wang, Philos Trans. of the Royal Society of London 339555 (1992).

[7] M.D. Kluge, D. Wolf, J.F. Lutsko and S.R. Phillpot, J. Appl. Phys. 672370 (1990).

[8] J.L. Bassani, V. Vitek and I. Alber, Acta Metall. mater. 40 S307 (1992).

[9] J.L. Bassani and J. Qu, in Metal-Ceramic Interfaces, edited by M. Rühle, A.G. Evans, M.F. Ashby, J.P. Hirth (Pergamon Press, Oxford, 1990), pp. 401-406.

[10] P. Peralta, L. Llanes, J. Bassani and C. Liard, Phil. Mag. A 70219 (1994).

[11] A.G. Marinopoulos, V. Vitek and J.L. Bassani, Phys. Stat. Sol. (a) 166453 (1998).

[12] M.J. Hÿtch, E. Snoeck and R. Kilaas, Ultramicroscopy 74131 (1998).

[13] P.B. Hirsch, A. Howie and M.J. Whelan, Philos. Trans. of the Royal Society of London Series A 252499 (1960).

[14] J. Thibault-Desseaux, J.-L. Putaux, A. Bourret and H.O.K. Kirchner, J. Phys. France 50 2525 (1989).

[15] HREM Research, online at: www.hremresearch.com.

[16] M.J. Hÿtch and T. Plamann, Ultramicroscopy 87199 (2001).

[17] F.R.N. Nabarro, Theory of Crystal Dislocations (Oxford University Press, Oxford, 1967).

[18] M.M.J. Treacy, J.M. Gibson and A. Howie, Philos. Mag. A 51389 (1985).

[19] H. Hashimoto and M. Mannami, Acta Cryst. 13363 (1960).

[20] A. Bourret, J. Desseaux and A. Renault, Phil. Mag. A 451 (1982).

[21] A.H. King and D.A. Smith, Acta Cryst. A 36335 (1980). 
[22] K.L. Merkle, R. Csencsits, K.L. Rynes, J.P. Withrow and P.A. Stadelmann, J. Electron Microscopy 190204 (1998).

[23] T. Xu and L. Zheng. Philos. Mag. Lett. 84225 (2004).

[24] J.Q. Su, M. Demura and T. Hirano. Acta. Mater. 512505 (2003).

[25] M. Haider, S. Uhlemann, E. Schwan, H. Rose, B. Kabius, K. Urban, Nature 392768 (1998). 
Tables

\begin{tabular}{|c|c|c|c|c|c|c|c|c|c|c|c|}
\hline \multicolumn{3}{|c|}{ Orientation } & \multicolumn{7}{c|}{ Stiffness tensor (GPa) } \\
\hline & $\mathrm{i}$ & $\mathrm{j}$ & $\mathrm{k}$ & $\mathrm{c}^{\prime}{ }_{11}$ & $\mathrm{c}^{\prime}{ }_{22}$ & $\mathrm{c}^{\prime}{ }_{66}$ & $\mathrm{c}^{\prime}{ }_{12}$ & $\mathrm{c}^{\prime}{ }_{13}$ & $\mathrm{c}^{\prime}{ }_{23}$ & $\mathrm{c}^{\prime}{ }_{16}$ & $\mathrm{c}^{\prime}{ }_{26}$ \\
\hline $\mathrm{Si}$ & {$[100]$} & {$[010]$} & {$[001]$} & 165.7 & & 79.6 & 63.9 & & & 0 & 0 \\
\hline $\mathrm{Si}$ & $\frac{1}{\sqrt{2}[110]}$ & {$[001]$} & $\frac{1}{\sqrt{2}}[1 \overline{1} 0]$ & 194.4 & 165.7 & 79.6 & 63.9 & 50.9 & 63.9 & 0 & 0 \\
\hline
\end{tabular}

Table 1: stiffness tensor components for silicon in the cubic axes [17] and for the orientations of the experimental image. 


\begin{tabular}{|c|c|c|c|c|c|c|c|l|l|l|l|}
\hline & \multicolumn{3}{|c|}{ Orientation } & \multicolumn{7}{c|}{ Stiffness tensor (GPa) } \\
\hline & $\mathrm{i}$ & $\mathrm{j}$ & $\mathrm{k}$ & $\mathrm{c}^{\prime}{ }_{11}$ & $\mathrm{c}^{\prime}{ }_{22}$ & $\mathrm{c}^{\prime}{ }_{66}$ & $\mathrm{c}^{\prime}{ }_{12}$ & $\mathrm{c}^{\prime}{ }_{13}$ & $\mathrm{c}^{\prime}{ }_{23}$ & $\mathrm{c}^{\prime}{ }_{16}$ & $\mathrm{c}^{\prime}{ }_{26}$ \\
\hline $\mathrm{Ge}$ & {$[100]$} & {$[010]$} & {$[001]$} & 128.9 & & 67.1 & 48.3 & & & 0 & 0 \\
\hline $\mathrm{Ge}$ & $\frac{1}{\sqrt{3}}[111]$ & $\frac{1}{\sqrt{6}}[\overline{1} \overline{1} 2]$ & $\frac{1}{\sqrt{2}}[1 \overline{1} 0]$ & 164.6 & 155.7 & 49.2 & 30.4 & 21.5 & 48.3 & 0.0 & -12.6 \\
\hline $\mathrm{Ge}$ & $\frac{1}{\sqrt{3}}[11 \overline{1}]$ & $\frac{1}{\sqrt{6}}[112]$ & $\frac{1}{\sqrt{2}}[1 \overline{1} 0]$ & 164.6 & 155.7 & 49.2 & 30.4 & 21.5 & 48.3 & 0.0 & 12.6 \\
\hline
\end{tabular}

Table 2: stiffness tensor components for germanium in the cubic axes and for the orientations of crystals $I$ and $I I$. 


\section{Figure Captions}

Figure 1: Geometric phase analysis of a matrix dislocation in silicon: (a) HRTEM image taken on a JEOL 200CX electron microscope operating at $200 \mathrm{kV}$ of a pure edge dislocation with Burgers vector $\mathbf{b}=1 / 2[110]$ seen end-on in [1 $\overline{1} 0]$ orientation; (b) in-plane displacement field measured experimentally by GPA and theoretical displacement field calculated from linear anisotropic elastic theory, $x$-axis parallel to Burgers vector, and spatial resolution 2-3 nm.

Figure 2: In-plane strain tensor components (a-c) and rigid-body rotation (d) measured experimentally (top) and determined theoretically (bottom). Contours every $0.5 \%$ from $-2.5 \%$ to $+2.5 \%$ strain, and every $0.5^{\circ}$ from $-2.5^{\circ}$ to $+2.5^{\circ}$ rotation (anticlockwise positive).

Figure 3: Strain tensor determined from linear anisotropic elastic theory assuming plane strain conditions (a-d) measured experimentally (top) and determined theoretically (bottom). Contours every $1 \mathrm{GPa}$ from $-5 \mathrm{GPa}$ to $+5 \mathrm{GPa}$.

Figure 4: Difference between experimental and theoretical $\varepsilon_{\mathrm{xx}}$ strain component (see Figure 2a). Contours every $0.5 \%$ from $-2.5 \%$ to $+2.5 \%$ strain.

Figure 5: Principal stress field component, $\sigma_{\mathrm{xx}}$, parallel to Burgers superimposed on original HRTEM image.

Figure 6: HRTEM image taken on a JEOL 200CX electron microscope operating at 200kV of a $\Sigma 3(111)$ coherent twin boundary in germanium. At centre is a dislocation with edge component $1 / 4[112]^{I}$ (referring to the lattice of grain $I$ ), corresponding to a $60^{\circ}$ matrix dislocation constricted in the boundary plane. Coordinates: $x$-axis perpendicular and $y$-axis parallel to boundary plane. 
Figure 7: In-plane strain tensor components (a-c) and rigid-body rotation (d) measured experimentally (top) and determined theoretically for an isolated matrix $60^{\circ}$ dislocation (bottom). Contours every $0.2 \%$ from $-2 \%$ to $+2 \%$ strain, and every $0.2^{\circ}$ from $-2^{\circ}$ to $+2^{\circ}$ rotation (anticlockwise positive). Experimental maps (b-d) constructed piece-wise from grains I and II, see text for details.

Figure 8: Difference between experimental $\varepsilon_{\mathrm{Xx}}$ strain component and theoretical values for an isolated dislocation (see Figure $7 \mathrm{a}$ ). Contours every $0.2 \%$ from $-2 \%$ to $+2 \%$ strain.

Figure 9: Principal strain field component, $\varepsilon_{\mathrm{xx}}$, parallel to Burgers superimposed on original HRTEM image.

Figure 10: Schematic model of grain boundary elastic response, assuming that a slab of material at grain boundary may have different elastic properties to the bulk. The presence of a dislocation applies compressive (above core) and tensile stresses (below core) to boundary producing localised strains.

Figure 11: Local stresses and strains across twin boundary plane: (a) $\varepsilon_{\mathrm{xx}}$ as a function of distance from dislocation core, above (negative) and below (positive), measured at the boundary (red), in the matrix (green), and from isotropic elastic theory (blue); (b) $\sigma_{\mathrm{xx}}$ as a function of distance from dislocation core, in the matrix (green), and from isotropic elastic theory (blue). Matrix values measured $3 \mathrm{~nm}$ from boundary on either side and averaged to provide an estimate of stresses at boundary plane. 


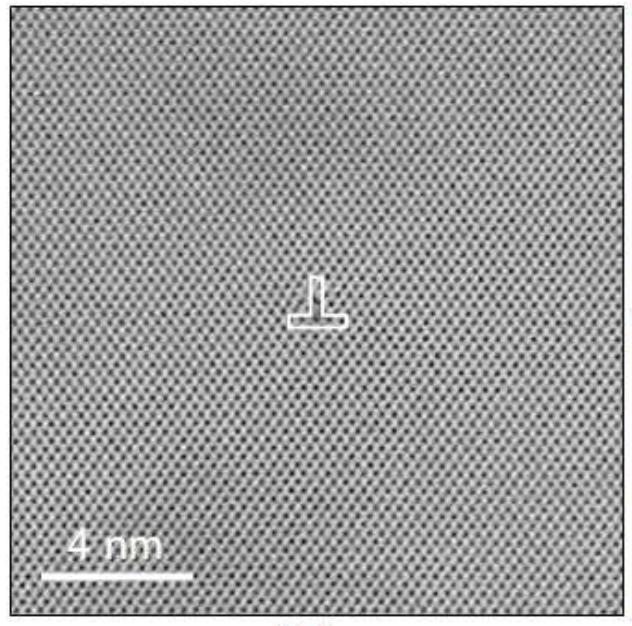

(a)

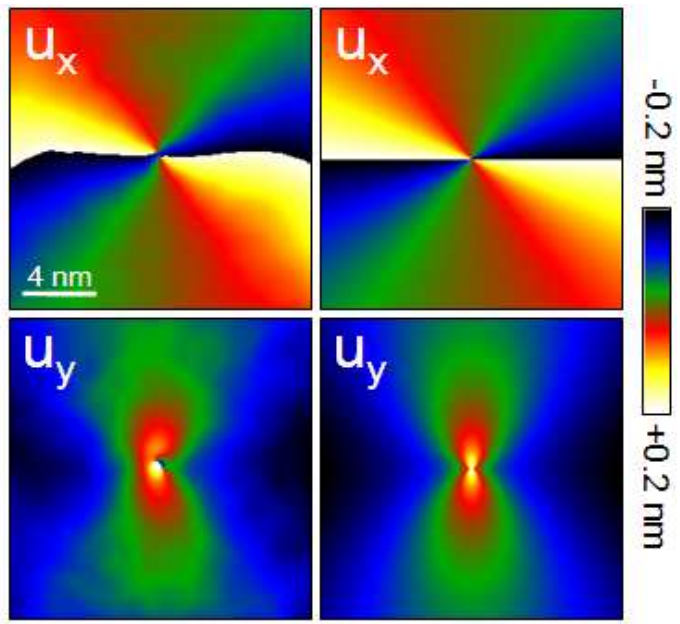

(b)

Figure 1

$186 \times 97 m m$ (96 x 96 DPI) 


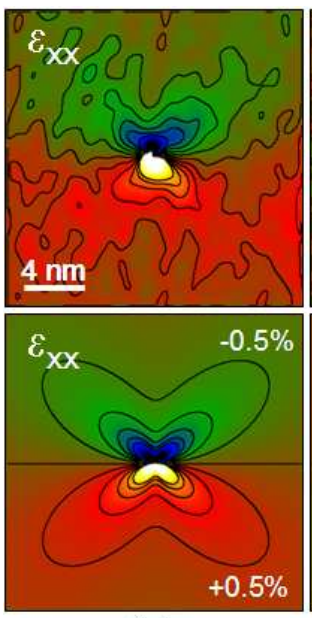

(a)
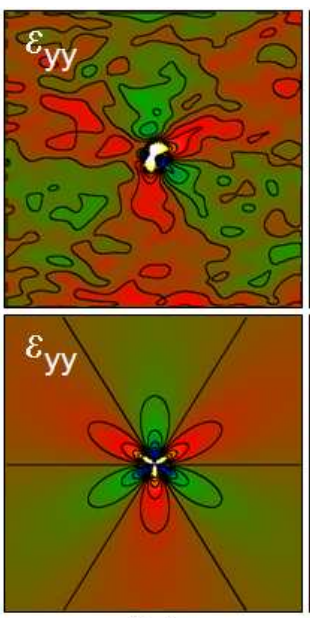

(b)
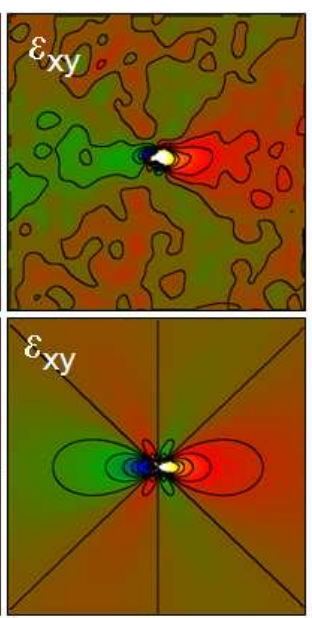

(c)

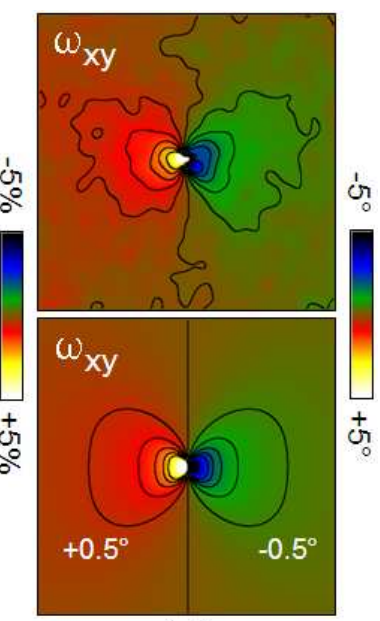

(d)

Figure 2

$230 \times 115 \mathrm{~mm}(96 \times 96 \mathrm{DPI})$ 


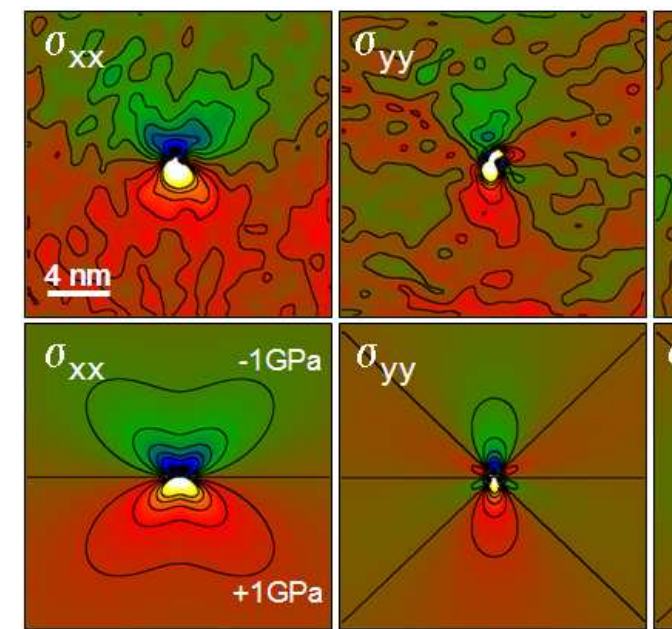

(a) (b)

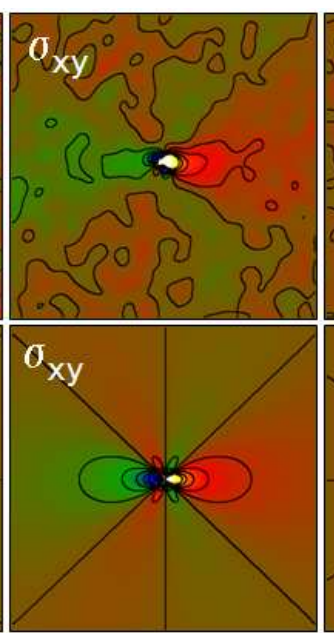

(c)

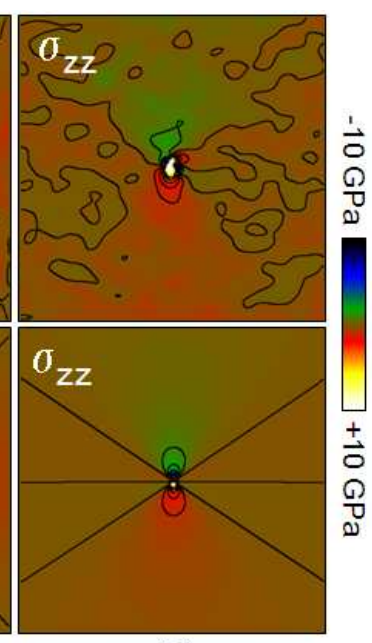

(d)

$223 \times 116 \mathrm{~mm}(96 \times 96 \mathrm{DPI})$ 


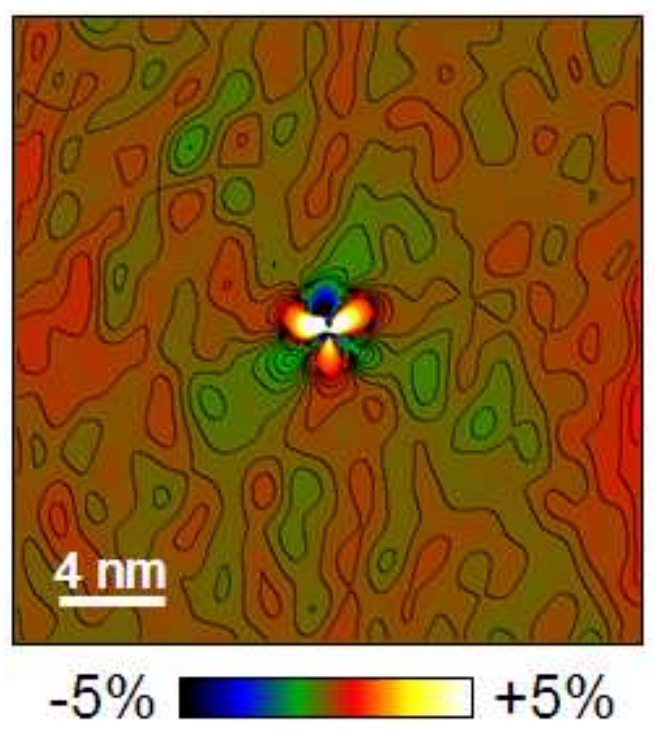

Figure 4 $65 \times 73 \mathrm{~mm}(96 \times 96 \mathrm{DPI})$ 


1
2
3
4
5
6
7
8
9
10
11
12
13
14
15
16
17
18
19
20
21
22
23
24
25
26
27
28
29
30
31
32
33
34
35
36
37
38
39
40
41
42
43
44
45
46
47
50
58
59
50
52
53
50

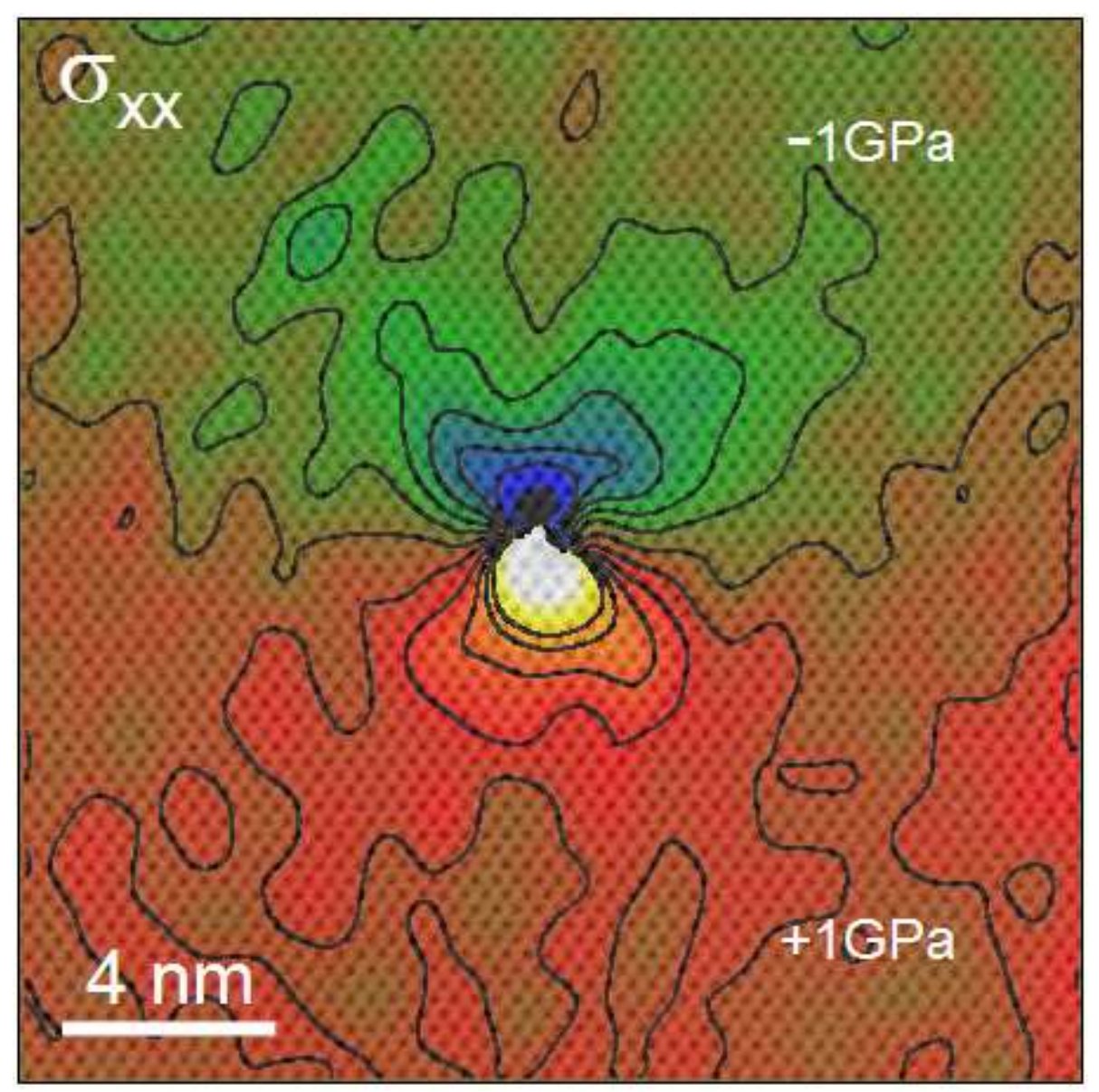

Figure 5 $120 \times 120 \mathrm{~mm}$ (96 x 96 DPI) 


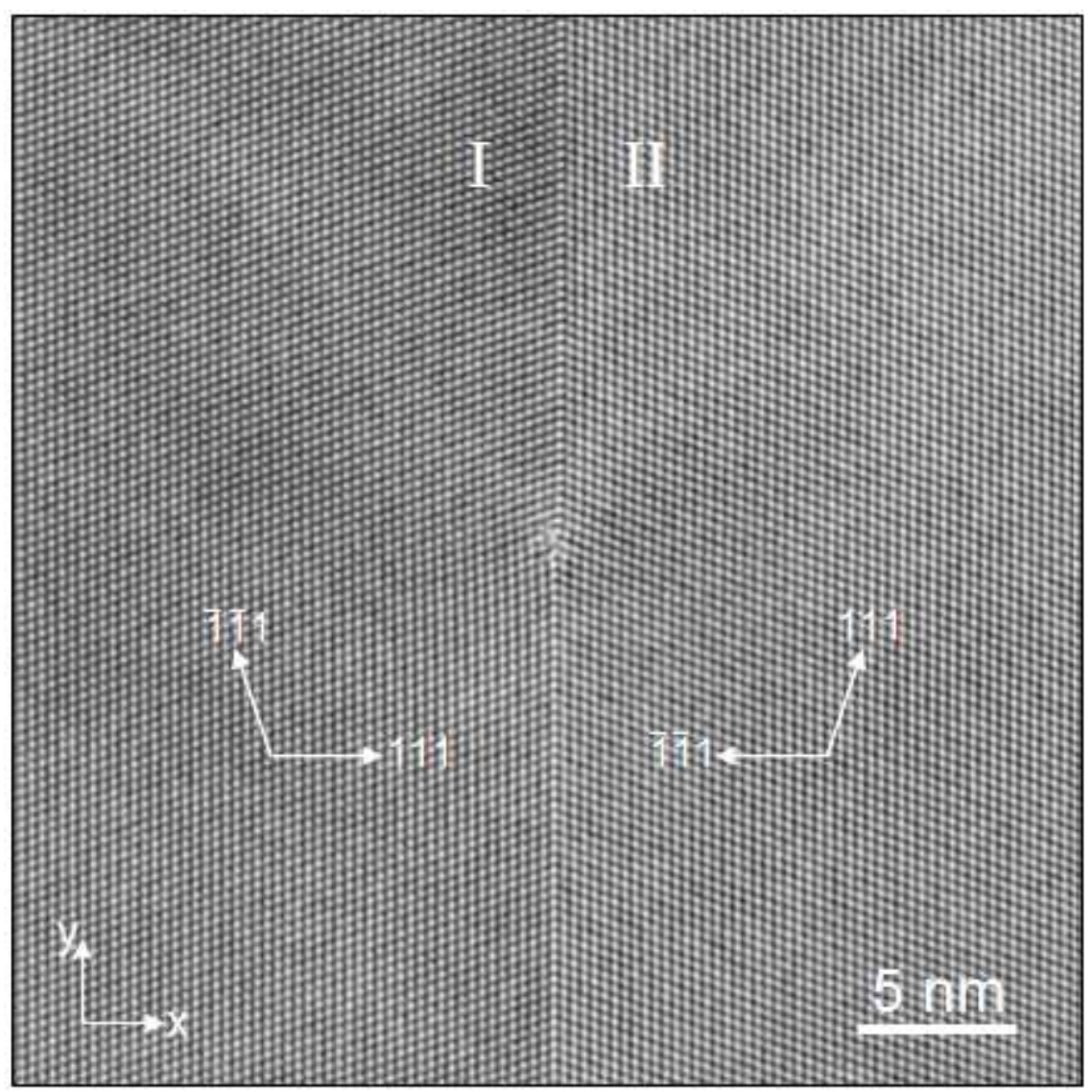

Figure 6

$115 \times 115 \mathrm{~mm}(96 \times 96 \mathrm{DPI})$ 


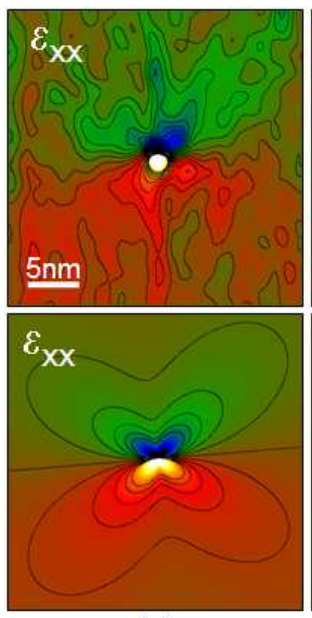

(a)

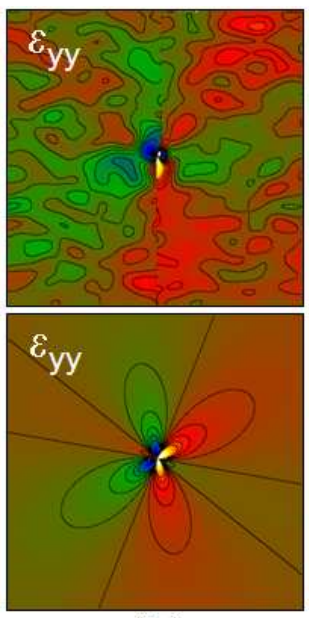

(b)

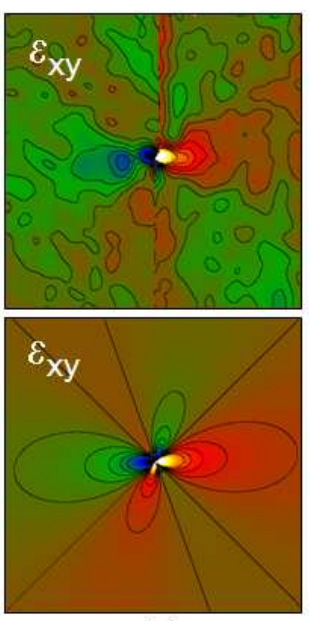

(c)

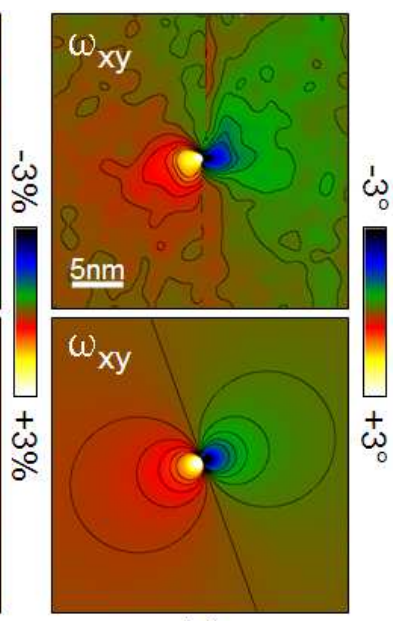

(d)

Figure 7

$227 \times 114 \mathrm{~mm}(96 \times 96 \mathrm{DPI})$ 


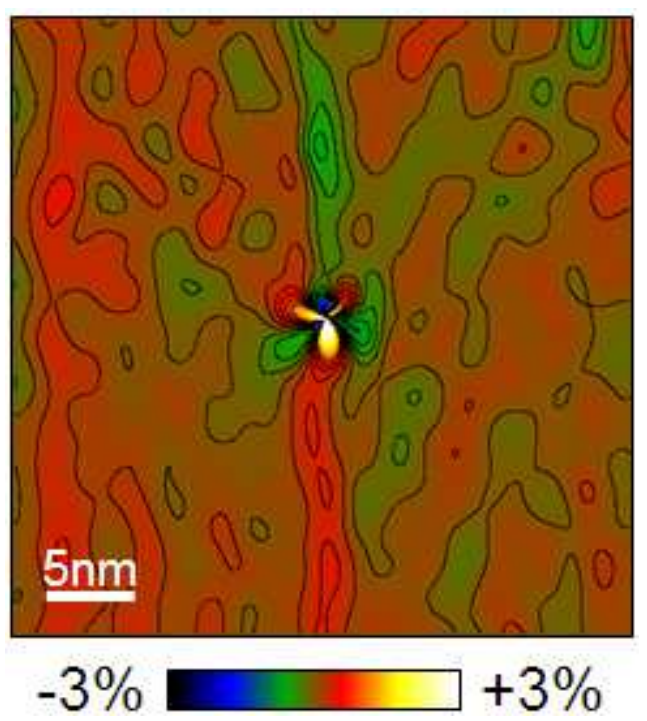

Figure 8 $64 \times 71 \mathrm{~mm}(96 \times 96$ DPI $)$ 


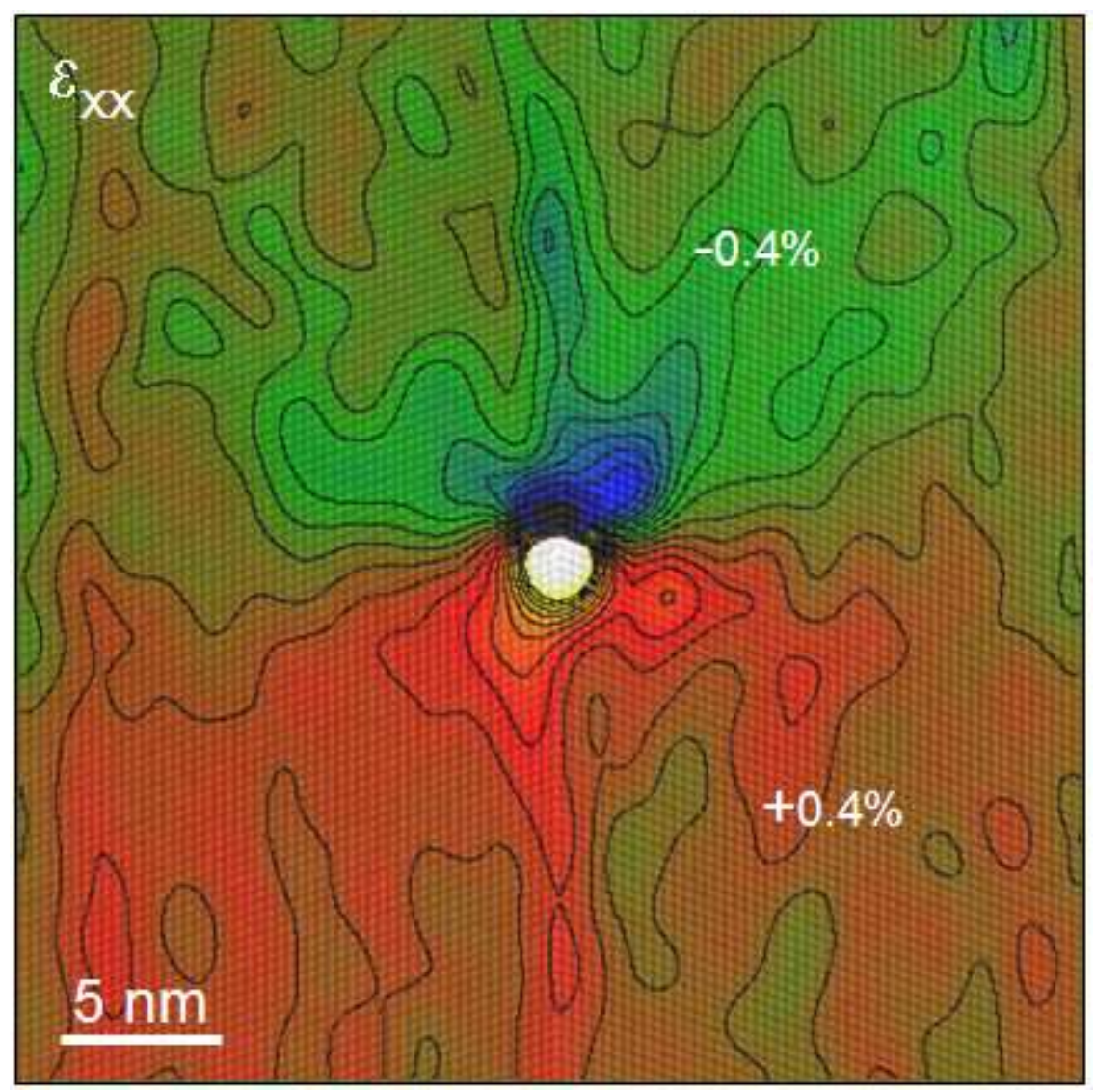

Figure 9

$114 \times 113 \mathrm{~mm}(96 \times 96 \mathrm{DPI})$ 


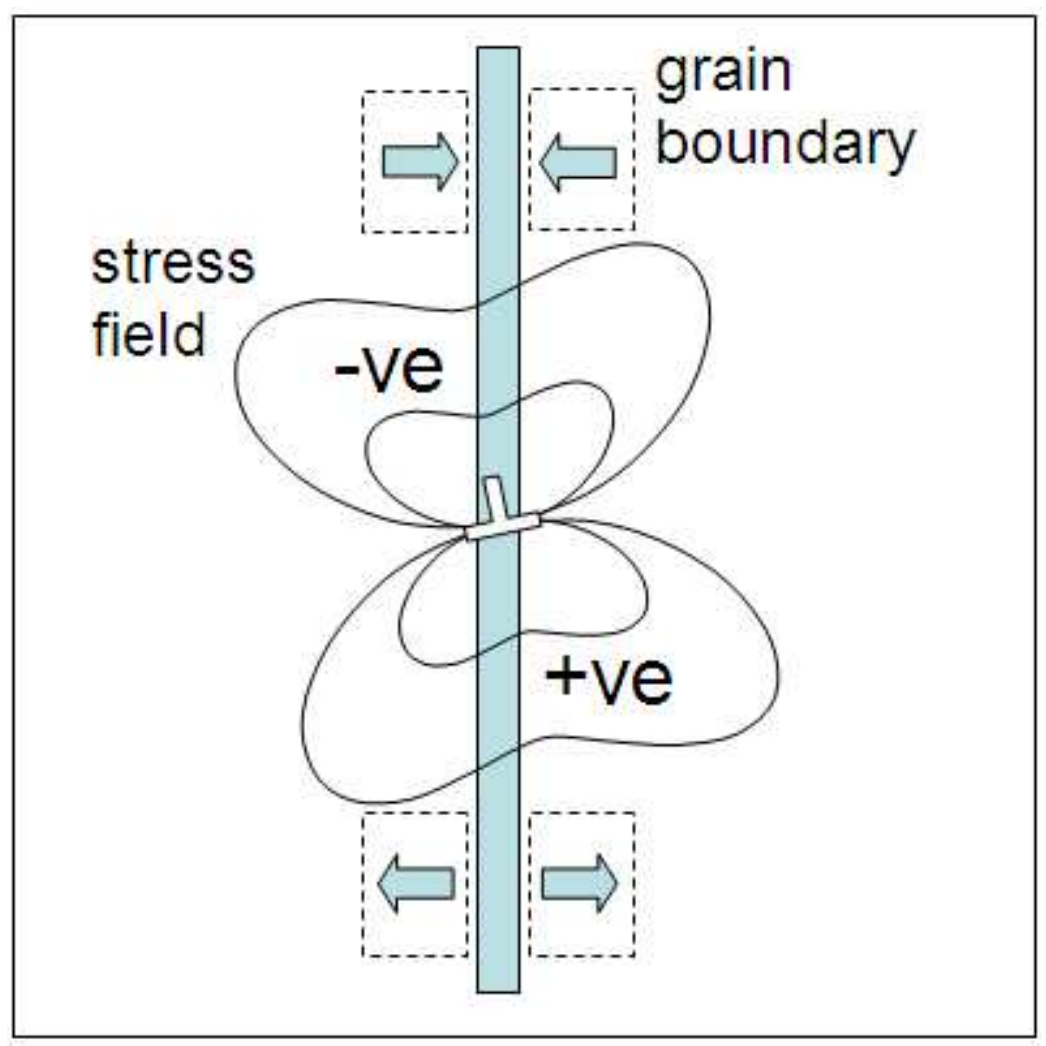

Figure 10

$105 \times 105 \mathrm{~mm}(96 \times 96 \mathrm{DPI})$ 

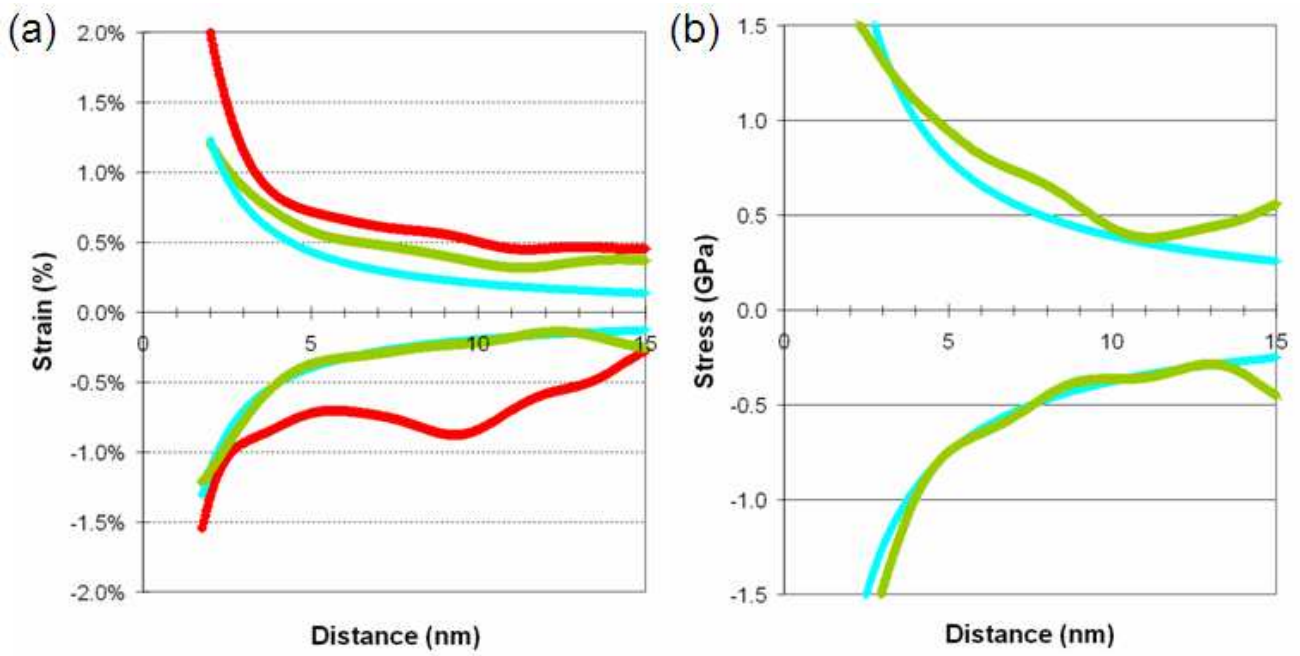

Figure 11

$200 \times 101 \mathrm{~mm}(96 \times 96$ DPI $)$ 